\title{
The Absorption of Gravitational Radiation by a Dissipative Fluid
}

\author{
J. Madore \\ Laboratoire de Physique Théorique, Institut Henri Poincaré, Paris, France
}

Received December 11, 1972

\begin{abstract}
An expression is found in the eikonal approximation for the gravitational radiation absorbed by a dissipative fluid.
\end{abstract}

\section{Introduction}

It is our purpose here to derive an expression for the damping of gravitational radiation as it passes through a dissipative fluid. We shall also calculate the entropy production in the fluid due to the passage of the radiation and verify the thermodynamical law $d S=d Q / T$ for a closed system.

We shall find that in the high frequency limit, the radiation is absorbed in a characteristic time $\eta^{-1}$ where $\eta$ is the shear viscosity. (We set $c=1$, $8 \pi G=1$.) This confirms a result of Hawking [1] in a more general context.

The eikonal approximation [2-4] is used. The notation is that of Ref. [4].

\section{Notation}

The signature of the metric is chosen to be -2 . We suppose that in some coordinate system the components of the metric describing the radiation may be written as the sum of two terms: $\tilde{g}_{\mu \nu}=g_{\mu \nu}+\varepsilon h_{\mu \nu}$. The components $g_{\mu \nu}$ of the background metric are relativity slowly varying functions with a characteristic length $L$. The potential $h_{\mu \nu}$ of the radiation depends explicitly on the point in space-time as well as on a phase function $\omega \phi . \omega$ is a constant with the dimension of inverse length. The derivative of $h_{\mu v}$ is given therefore by

where

$$
\partial_{\lambda} h_{\mu \nu}=\omega \xi_{\lambda} \dot{h}_{\mu \nu}+h_{\mu \nu, \lambda}
$$

$$
\xi_{\lambda}=\partial_{\lambda} \phi, \quad \dot{h}_{\mu \nu}=\frac{\partial h_{\mu v}}{\partial(\omega \phi)}, \quad h_{\mu v, \lambda}=\left(\partial_{\lambda} h_{\mu \nu}\right)_{\phi=\text { const }} .
$$


We suppose that $\left|h_{\mu \nu, \lambda}\right| \sim L^{-1}\left|h_{\mu \nu}\right|$. Define $\delta=1 / \omega L$. The eikonal approximation is $\delta \ll 1$. We suppose also that $\varepsilon \ll \delta$ and we neglect terms quadratic in $\varepsilon$. This means in particular that we neglect the energy of the wave. We set

$$
h_{\mu v ; \lambda}=\left(D_{\lambda} h_{\mu \nu}\right)_{\phi=\text { const }} .
$$

We define $r$ by $\frac{d x^{\alpha}}{d r}=\xi^{\alpha}$ and we set ${ }_{;} \xi^{\alpha}=\delta / \delta r$.

The energy-momentum tensor of a dissipative fluid is [5-9]

$$
T_{\mu \nu}=w u_{\mu} u_{v}-p g_{\mu \nu}+\eta \sigma_{\mu v}+\zeta \theta \pi_{\mu \nu}+\kappa u_{(\mu} q_{v)},
$$

where $w$ is the enthalpy per unit volume, $p$ is the pressure, $\eta, \zeta$ are the coefficients of shear and bulk viscosity and $\kappa$ is the coefficient of thermal conductivity.

$$
\pi_{\mu \nu}=g_{\mu \nu}-u_{\mu} u_{\nu}
$$

is the projection on the 3 -space normal to the flow.

$$
\sigma_{\mu \nu}=\pi_{\mu}^{\alpha} \pi_{\nu}^{\beta} u_{(\alpha ; \beta)}-\frac{2 \theta}{3} \pi_{\mu \nu}, \quad \theta=u_{; \alpha}^{\alpha}
$$

are the shear and dilatation rates.

is the heat flow vector.

$$
q_{\mu}=\pi_{\mu}^{\alpha} T_{, \alpha}-T u_{\mu ; v} u^{v}
$$

The coefficients $\eta, \zeta, \kappa$ satisfy

$$
0 \gtrless \eta, \zeta, \kappa T \ll L w .
$$

The entropy flow vector is

$$
S^{\alpha}=n \sigma u^{\alpha}+\frac{\kappa}{T} q^{\alpha},
$$

where $n$ is the particle number density and $\sigma$ is the entropy per particle. The equation of continuity, $u_{\mu} T_{; v}^{\mu v}=0$, and the equation of particle number conservation, $\left(n u^{\mu}\right)_{; \mu}=0$ yield

$$
S_{; x}^{\alpha}=\frac{\eta}{2 T} \sigma_{\alpha \beta} \sigma^{\alpha \beta}+\frac{\zeta \theta^{2}}{T}-\frac{\kappa}{T^{2}} q_{\alpha} q^{\alpha} \geqq 0 .
$$

In the presence of the wave all of the quantities which describe the fluid will be perturbed from their former value by a small amount of the order of $\varepsilon: \tilde{u}_{\mu}=u_{\mu}+\varepsilon u_{\mu}^{\prime}, \tilde{w}=w+\varepsilon w^{\prime}, \tilde{p}=p+\varepsilon p^{\prime}$,

$$
\tilde{T}=T+\varepsilon T^{\prime}, \tilde{\eta}=\eta+\varepsilon \eta^{\prime}, \tilde{\zeta}=\zeta+\varepsilon \zeta^{\prime}, \tilde{\kappa}=\kappa+\varepsilon \kappa^{\prime} .
$$

The perturbations in $\eta, \zeta$ and $\kappa$ may be thought to be due to the presence of radiative viscosity [10]. We shall suppose that they do not depend of the frequency of the radiation. We shall write all perturbed quantities in this form: $\tilde{\pi}_{\mu \nu}=\pi_{\mu \nu}+\varepsilon \pi_{\mu \nu}^{\prime}$ etc. 


\section{Energy Absorption}

The basic equations are the Einstein field equations for the perturbed and unperturbed metrics:

$$
\tilde{G}_{\mu \nu}=-\tilde{T}_{\mu \nu}, \quad G_{\mu \nu}=-T_{\mu \nu},
$$

and the conservation laws in the perturbed and unperturbed metrics:

$$
\tilde{D}^{\mu} \tilde{T}_{\mu \nu}=0, \quad T_{\mu v}{ }^{{ }}{ }=0 .
$$

We shall discuss the latter first. Before proceeding however, it is preferable to impose a gauge condition.

Define $\psi_{\lambda \mu}$ by

$$
\psi_{\lambda \mu}=h_{\lambda \mu}-\frac{h}{2} g_{\lambda \mu}, \quad h=h_{\alpha}^{\alpha} .
$$

We shall suppose in what follows that the approximate de Donder condition holds

$$
D^{\lambda} \psi_{\lambda \mu}=O(\delta / L) \text {. }
$$

Under a coordinate transformation of the form

$$
x^{\mu} \rightarrow x^{\mu}+\varepsilon / \omega f^{\mu},
$$

the potential transforms as

$$
h_{\mu \nu} \rightarrow h_{\mu \nu}-\dot{f}_{(\mu} \xi_{v)}-\frac{1}{\omega} f_{(\mu ; v)} .
$$

The gauge condition (3.3) admits the restricted set of coordinate transformations given by (3.4) with $f_{\mu}$ satisfying

$$
2 \frac{\delta f_{\mu}}{\delta r}+\xi_{; \lambda}^{\lambda} f_{\mu}=o(\delta / L) \text {. }
$$

We have from (3.3), $\psi_{\lambda \mu} \xi^{\mu}=o(\delta)$.

It is also conveniant to suppose that the hypersurfaces of constant phase are null:

$$
\xi^{2}=o\left(\delta^{2}\right) .
$$

This can in fact be proven from the field Eqs. (3.1), but it would lengthen somewhat the calculations without adding anything essentially new.

The components of the perturbed matter tensor are

where

$$
\tilde{T}_{\mu v}=T_{\mu v}+\varepsilon T_{\mu v}^{\prime},
$$

$$
\begin{aligned}
T_{\mu v}^{\prime}= & w^{\prime} u_{\mu} u_{v}+w u_{(\mu} u_{v)}^{\prime}-p^{\prime} g_{\mu v}-p h_{\mu v}+\eta \sigma_{\mu v}^{\prime}+\eta^{\prime} \sigma_{\mu v}+\zeta \theta^{\prime} \pi_{\mu v} \\
& +\zeta \theta \pi_{\mu v}^{\prime}+\kappa u_{(\mu}^{\prime} q_{v)}+\kappa u_{(\mu} q_{v)}^{\prime}+\zeta^{\prime} \theta \pi_{\mu v}+\kappa^{\prime} u_{(\mu} q_{v)} .
\end{aligned}
$$

Even when we neglect terms of order $\delta$, this expression for $T_{\mu \nu}^{\prime}$ is rather complicated. Using (2.5) however it may be considerably simplified. 
In the adiabatic limit the conservation Eqs. (3.2) yield

$$
w u_{\alpha} \xi^{\alpha} \dot{u}_{\mu}^{\prime}+\left(\dot{w}^{\prime} u_{\alpha} \xi^{\alpha}+w \dot{u}_{\alpha}^{\prime} \xi^{\alpha}\right) u_{\mu}-\left(\dot{p}^{\prime}+\frac{w}{2} \dot{h}_{\alpha \beta} u^{\alpha} u^{\beta}\right) \xi_{\mu}=o(\delta / L) .
$$

Therefore

$$
u_{\mu}^{\prime}=\frac{h_{\alpha \beta} u^{\alpha} u^{\beta}}{u_{\alpha} \xi^{\alpha}} \xi_{\mu}+o(\delta), \quad p^{\prime}, w^{\prime}=o\left(\delta / L^{2}\right) .
$$

Since $T^{\prime}=\left(\frac{\partial T}{\partial p}\right)_{s} p^{\prime}$, we have also

$$
T^{\prime}=o(\delta L)
$$

Placing these values for $u_{\mu}^{\prime}, p^{\prime}, w^{\prime}, T^{\prime}$ in the expressions for $\sigma_{\mu \nu}^{\prime}, \theta^{\prime}, q_{v}^{\prime}$ in (3.7) and applying once more the conservation Eqs. (3.2) we find that, to first order in $\eta, \zeta, \kappa T$, we have

$$
u_{\mu}^{\prime}=o(1), \quad p^{\prime}, w^{\prime}=o\left(L^{-2}\right) .
$$

Therefore, to first order in $\eta, \zeta, \kappa T$, we find the following expression for $T_{\mu \nu}^{\prime}$ :

$$
T_{\mu \nu}^{\prime}=\omega\left[\eta\left(u_{\alpha} \xi^{\alpha} \dot{h}_{\mu \nu}-\xi_{(\mu} \dot{h}_{v) \alpha} u^{\alpha}+\frac{\dot{h}_{\alpha \beta} u^{\alpha} u^{\beta}}{u_{\alpha} \xi^{\alpha}} \xi_{\mu} \xi_{\nu}\right)+o(\delta / L)\right] .
$$

If we write the components of the perturbed Einstein tensor as

$$
\tilde{G}_{\mu \nu}=G_{\mu \nu}+\varepsilon G_{\mu \nu}^{\prime},
$$

then the field Eqs. (3.1) imply

$$
G_{\mu \nu}^{\prime}=-\omega\left[\eta\left(u_{\alpha} \xi^{\alpha} \dot{h}_{\mu \nu}-\xi_{(\mu} \dot{h}_{\nu) \alpha} u^{\alpha}+\frac{\dot{h}_{\alpha \beta} u^{\alpha} u^{\beta}}{u_{\alpha} \xi^{\alpha}} \xi_{\mu} \xi_{\nu}\right)+o(\delta / L)\right] .
$$

In the gauges (3.3) $G_{\mu \nu}^{\prime}$ is given by (This follows, for example, from [4], formulae (3.12).)

$$
G_{\mu \nu}^{\prime}=\frac{\omega^{2}}{2} \xi^{2} \ddot{\psi}_{\mu \nu}+\frac{\omega}{2}\left(2 \frac{\delta \dot{\psi}_{\mu \nu}}{\delta r}+\xi_{; \lambda}^{\lambda} \dot{\psi}_{\mu \nu}+o(\delta / L)\right) .
$$

Using (3.6) we have therefore

$2 \frac{\delta \psi_{\mu \nu}}{\delta r}+\xi_{; \lambda}^{\lambda} \psi_{\mu \nu}=-2 \eta\left(u_{\alpha} \xi^{\alpha} h_{\mu \nu}-\xi_{(\mu} h_{\nu) \alpha} u^{\alpha}+\frac{h_{\alpha \beta} u^{\alpha} u^{\beta}}{u_{\gamma} \xi^{\gamma}} \xi_{\mu} \xi_{\nu}\right)+o(\delta / L)$.

Taking the trace of this equation, we find the following conservation law for $h$ :

$$
\left(h^{2} \xi^{\lambda}\right)_{; \lambda}=o(\delta / L) .
$$

From (3.5) the remaining gauge freedom may be therefore used to put $h=0$. 
Eq. (3.15) yields then the following equation for $h_{\mu \nu}$ :

where

$$
2 \frac{\delta h_{\mu \nu}}{\delta r}+\xi_{; \lambda}^{\lambda} h_{\mu \nu}=-2 \eta\left(u_{\alpha} \xi^{\alpha} h_{\mu \nu}-X_{(\mu} \xi_{\nu}\right)+o(\delta / L),
$$

$$
X_{\mu}=h_{\mu \alpha} u^{\alpha}-\frac{\xi_{\mu}}{2 u_{\alpha} \xi^{\alpha}} h_{\alpha \beta} u^{\alpha} u^{\beta} .
$$

If we write explicitly the eikonal ansatz, giving $h_{\mu \nu}$ as the product of an amplitude $a$, a (possibly complex) polarization matrix $n_{\mu \nu}$ and a phase factor $e^{i \omega \phi}$ :

$$
h_{\mu \nu}\left(x^{\alpha}, w \phi\right)=a\left(x^{\alpha}\right) n_{\mu \nu}\left(x^{\alpha}\right) e^{i \omega \phi},
$$

with $\bar{n}_{\mu \nu} n^{\mu \nu}=1$, then neglecting terms of order $\delta$, we have a decay law for $a$ :

$$
\left(a^{2} \xi^{\lambda}\right)_{; \lambda}=-2 \eta a^{2} u_{\alpha} \xi^{\alpha}
$$

and a transport law for $n_{\mu \nu}$ :

$$
\frac{\delta n_{\mu \nu}}{\delta r}=\frac{\eta}{a} \xi_{(\mu} X_{v)}
$$

Eq. (3.20) may be rewritten

$$
\frac{d \alpha}{d r}=\eta u_{\alpha} \xi^{\alpha}
$$

if we set $a=e^{-\alpha} b$ with $\left(b^{2} \xi^{\lambda}\right) ; \lambda=0$.

The function $\phi$ may be normalized such that at any particular point in the fluid $u_{x} \xi^{\alpha}=1 ; \omega$ is then the frequency of the wave at that point. We may therefore say that the radiation is absorbed in a characteristic time $\eta^{-1}$.

The vector $X_{\mu}$ does not seem to have any obvious physical meaning. We remark however that it is uniquely defined by the requirement that the right-hand side of Eq. (3.17) be normal to $u^{\mu}$.

\section{Entropy Production}

Designate by $\bar{f}$ the mean value of a function $f$ taken over a volume whose dimensions are large with respect to $1 / \omega$ but small with respect to $L$. The average value of a function which depends linearly on the small perturbations due to the passage of the radiation, will vanish.

From formula (2.7) a straightforward calculation yields therefore to first order in $\eta, \zeta, \kappa T$

$$
\overline{\tilde{D}_{\alpha} \tilde{S}^{\alpha}-S_{; \alpha}^{\alpha}}=\frac{\varepsilon^{2} \omega^{2} \eta}{4 T}\left(\left(u_{\alpha} \xi^{\alpha}\right)^{2} a^{2}+o(\delta)\right) .
$$


The average energy-momentum tensor of the wave is [2]

From (3.20)

$$
\bar{\tau}_{\mu v}=\frac{(\varepsilon \omega a)^{2}}{8}\left(\xi_{\mu} \xi_{v}+o(\delta)\right) .
$$

$$
\bar{\tau}_{; v}^{\mu \nu}=-\eta \frac{(\varepsilon \omega a)^{2}}{4}\left(u_{x} \xi^{x} \xi^{\mu}+o(\delta)\right) .
$$

We have therefore, neglecting terms of order $\delta$,

$$
\overline{\tilde{D}_{\alpha} \tilde{S}^{x}-S_{; \alpha}^{x}}=-\frac{u_{\mu} \bar{\tau}_{; v}^{\mu v}}{T} .
$$

The quantity $-u_{\mu} \bar{\tau}_{; v}^{\mu v}$ is the average rate of energy transfer per unit volume from the radiation to the fluid. Since the fluid does no work, this represents the rate of increase in the heat content per unit volume $d Q$ of the fluid. The left-hand side of (4.4) in the entropy per unit volume $d S$ supplied to the fluid by the radiation per unit time. (4.4) is therefore a particular case of the Carnot-Clausius relation $d S=d Q / T$.

The ratio of $\overline{\tilde{D}_{\alpha} \tilde{S}^{\alpha}-S_{; \alpha}^{\alpha}}$ to $S_{; \alpha}^{\alpha}$ is of the order of magnitude of $(\varepsilon / \delta)^{2}$ which we have supposed to be much less than one. The amount of entropy produced in the fluid by the radiation is negligeable compared with the amount of entropy produced by ordinary dissipative effects.

\section{References}

1. Hawking, S. W.: Ap. J. 145, 544 (1966).

2. Isaacson, R. A.: Phys. Rev. 160, 1263, 1272 (1968).

3. Choquet, Y.: C.R.A.S. 258, 1089 (1964); Commun. math. Phys. 12, 16 (1969).

4. Madore, J.: Commun. math. Phys. 27. 291 (1972).

5. Eckhart, C.: Phys. Rev. 58, 919 (1940).

6. Landau, L. D., Lifshitz,E. M.: Fluid Mechanics. Oxford: Pergamon Press 1959.

7. Marle, C.: Ann. Inst. H. Poincaré 10, 67 (1969).

8. Israel, W: The Relativistic Boltzman Equation, in General Relativity, Papers in Honour of J. L. Synge. Oxford: Clarendon Press 1972.

9. Ehlers, J.: In General Relativity and Cosmology, (R. K. Sachs Ed.), New York: Academic Press 1971.

10. Weinberg, S.: Ap. J. 168, 175 (1971).

J. Madore

Laboratoire de Physique Théorique Institut Henri Poincaré 11 rue Pierre et Marie Curie F-75231 Paris Cedex 05, France 